\title{
Maternal and perinatal outcomes in pregnant women with SARS-CoV-2 infection: a retrospective observational study
}

Carmen lopez gorosabel ( $\nabla$ clgorosabel@hotmail.com )

Hospital Universitario Infanta Leonor: Hospital Infanta Leonor https://orcid.org/0000-0002-5733-0364 Soto-Sanchez Eva Maria Ibañez-Santamaria Ana Belen

Sanchez Estevez Beatriz Hernandez-Aguado Juan Jose

\section{Research Article}

Keywords: COVID-19, maternal outcome, neonatal outcome, obstetric outcomes, pregnancy, SARS-CoV-2

Posted Date: February 4th, 2022

DOl: https://doi.org/10.21203/rs.3.rs-1314631/v1

License: (c) (i) This work is licensed under a Creative Commons Attribution 4.0 International License.

Read Full License 


\section{Abstract \\ Objectives}

The aim of our study is to present the impact on maternal and perinatal outcomes of the COVID pandemic in our patients.

\section{Methods}

We retrospectively reviewed the medical records of all pregnant women from March to December 2020 in Infanta Leonor Universitary Hospital, in Madrid. Infected women during the 3rd trimester of pregnancy or at delivery time with SARS-CoV2 diagnosis composed infected group, and non-infected women with SARS-CoV2 composed non-infected group. The infection status was determined via RT-PCR of nasopharyngeal swab specimen.

\section{Results}

1262 non-infected pregnant women and 86 SARS-CoV2 positive pregnant women were admitted to our clinic during the study period. Within the group of infected pregnant women, they were 49 cases (56.98\%) symptomatic and 37 cases (43.02\%) asymptomatic. In the group of symptomatic infected women, the majority were mild cases (80.23\%). In the group of uninfected pregnant women, the main complication was gestational diabetes 14 (1.09\%) We did not find any complications during pregnancy in case group. Cesarean section was performed in $16.67 \%$ of the infected pregnant women and in $21.71 \%$ of the control group. Mean neonatal birth weight was $3.240 \mathrm{~g}$ in case group and $3.210 \mathrm{~g}$ in non-infected group. Neonatal admission to NICU was $9.52 \%$ in cases while in the control group was $1.19 \%$.

\section{Conclusion}

Infection does not worsen the prognosis of pregnancy in terms of low birth weight of the newborn, but is associated with a greater increase in neonatal admission in NICU.

\section{Introduction}

Since the beginning of the worldwide outbreak on December 31, 2019, over 183 million cases have been reported and mortality rate differs substantially among countries. Specifically, Spain has reported more than 3.800 .000 confirmed cases and 80.000 deaths from the beginning of the pandemic till September, 2021.(1)

The physiological changes(2) that occur in pregnancy make pregnant women a high-risk group in the COVID 19 pandemic(3). The evidence suggests that pregnancy does not increase susceptibility to Covid- 
19 and pregnancy but appears to worsen the clinical course of COVID-19 compared with non pregnant females of the same age (4). Although more than $90 \%$ infected pregnant women recover without hospitalization, rapid clinical deterioration can occur sometimes, and symptomatic pregnant patients appear to be an increased risk of severe disease and death compared with symptomatic non pregnant females of reproductive age (5) (6) (7). According to the currently available data, the risk factors for severe disease and death in these patients include $\geq 35$ years old, obesity, and preexisting medical comorbidities like hypertension or diabetes(8) (9) (10).

We aim is to know better the relationship between maternal infection and maternal and perinatal outcomes.

\section{Material And Methods}

This study is a retrospective cohort study of pregnant women registered in Infanta Leonor Universitary Hospital (Madrid, Spain) from March 1 to December 31 2020, whose delivery occurs in our hospital. All data was collected using the clinical notes in the general authorized hospital database. We recorded pregnant patients with and without confirmed COVID-19 diagnosis (which had a positive SARS-CoV-2 RTPCR of nasopharyngeal swabs)(11) following the recommendations from the Royal College of Obstetricians and Gynecologists, which suggest making a universal screening of all women admitted to maternity service. All the patients included in the study were controlled in our area and gave birth in our hospital.

The inclusion criteria in infected group were women who had a SARS-CoV-2 infection during the 3rd trimester of gestation or during their admission to delivery in the emergency department due to onset of labor or premature rupture of membranes (infection confirmed by means of SARS-CoV-2 positive RT-PCR of nasopharyngeal swabs) and the inclusion criteria in non-infected group was defined as having a negative RT-PCR at the 3rd trimester of pregnancy or at admission to delivery, and with no symptoms preor postpartum. In both groups, exclusion criteria were multiple pregnancy and delivery outside our Hospital.

Women information about demographic characteristics, comorbidities and obstetric history was extracted from the Hospital database. Age and race categories were following the classifications used by the CDC (Center for Disease Control and Prevention). Parity was defined using the records of previous births and divided into two categories: primiparous and multiparous. Information about maternal comorbidities like diabetes mellitus or hypertension was available in the diagnosis codes attached to the birth episode in each medical history. Also, more data was recorded from maternal medical history like: obstetric complications (preeclampsia, eclampsia), the need for prenatal steroid treatment for lung maturation with betamethisone, the disease severity scale, medical maternal complications (pneumonia or UCl admission), the inductions of labor, and the birth mode (unassisted vaginal delivery, instrumental vaginal delivery and cesarean delivery). Furthermore, data related to the newborn such as gestational age at the time of delivery, newborn weight, one minute and 5 minutes APGAR test, as well as neonatal 
resuscitation or admission to the ICU, were consulted in the newborn's medical history. The definitions of the different clinical and obstetric conditions were established by international criteria.

Description of the quantitative values was made through the descriptive statistics of the mean, the standard deviation, and the confidence interval for the mean. Since the data distributions may not follow a Gaussian distribution, other robust statistics such as the median and the interquartile range are also indicated, as well as the maximum and minimum values. Distributions of categorical variables have been described by means of absolute and percentage frequencies of the distribution. To detect statistically significant relationships between the categorical ones, Fisher's exact test will be used. To test statistically significant differences in continuous variables of scale, ratio or interval, the Student's $t$ test will be applied, using the Kruskall-Wallis test in the case of not fulfilling the assumptions for its application. Data were collected and managed using REDCap (Research Electronic Data Capture) tools, hosted at Ideas for Health Association, a secure web-based software platform designed to support data Capture for research studies (12).

The study was carried out following The Ethics Code of the World Medical Association (declaration of Helsinki) (13). The Institutional Investigation and Ethic Review Board of Infanta Leonor University Hospital (CEI-HUIL) approved the study (Code ILUH R 000-21). Given the characteristics of the study, informed consent of pregnant patients is not required.

\section{Results}

During the study period, from March 1 to December 31, 2020; a total of 1.348 patients were recorded: 86 pregnant women infected by COVID 19 and 1.262 non-infected.

Demographic features and clinical characteristics of all patients are shown in Table 1.

The mean maternal age in non-infected group was 31.5 years (26-36) and in infected group was 30 years (28.3-31.2). Secondly, regarding ethnicity, among infected women group, 29 patients were from Spain 29 $(34.5 \%)$ while $55(65.5 \%)$ were from outside of our country. In the third place, about parity in the infected group there was $48.3 \%$ nulliparous and $51.7 \%$ of multiparous and in the non-infected group, there was $44.4 \%$ of nulliparous and $55.6 \%$ multiparous.

In infected group there was $4.64 \%$ (4 cases) thyroid disfunction, $1.16 \%$ (1 case) pulmonary comorbidities, $2.32 \%$ ( 2 cases) hematologic comorbidities, $10.47 \%$ (9 cases) obesity, $4.65 \%$ (4 cases) hypertension disease and $5.81 \%$ (5 cases) pregestacional diabetes; and in non-infected group there was $0.95 \%$ (12) tyroid disfunction, $0.39 \%$ (5 cases) pulmonary comorbidites, $0.85 \%$ (13 cases) hematologic comorbidites, $6.34 \%$ (80 cases) obesity, $1.58 \%$ (22 cases) hypertension disease and $1.58 \%$ (20 cases) pregestacional diabetes.

Of the 86 positive pregnant women during the 3rd trimester of pregnancy or at delivery, $57 \%$ (49) were symptomatic while $43 \%(37)$ were asymptomatic. 
Symptomatic pregnant women were classified into three groups: mild $80.2 \%(69)$, moderate $11.6 \%$ (10) and severe $8.1 \%(7)$ being this last group of those pregnant women complicated with pneumonia.

Clinical manifestations in infected women occurred in $56.9 \%$ being ageusia the most frequent symptom present in $100 \%$ of cases, followed in second place by fever with $36 \%$. Less frequent was headache $(16.3 \%)$ and myalgias (11.6\%). Mean gestational age at diagnosis was 38 weeks (36.1-37.8) and the mean duration of COVID 19 infection was 15 days (15.6-17.7). These characteristics of infected pregnant women are shown in Table 2.

\section{Pregnancy outcomes}

Obstetric and neonatal outcomes in SARS-COV-2 infected pregnant women are shown in Table 3.

Regarding comorbidities $9.29 \%$ (8) in infected group were obese while only 5.9\% (73 patients) in noninfected group; $4.6 \%$ (4) of infected women had chronic hypertension versus $1 \%$ (13) of non-infected group; and $4.65 \%$ (4) had pregestational diabetes among infected women versus $0.9 \%$ (11) in noninfected group.

We did not observe any complications such as preeclampsia, gestational diabetes or fetal growth restriction in infected group. Nevertheless, in non-infected group, we found gestational diabetes in $1.1 \%$ (14) and fetal growth restriction in $1.01 \%$ (13).

About the way of labor in infected group, $83.3 \%$ of the deliveries were vaginal deliveries (59 unassisted delivery, 11 instrumental delivery) and $16.7 \%$ were by cesarean section (14). On the other hand, in noninfected group, we found $77.9 \%$ of vaginal deliveries (863 unassisted delivery, 120 instrumental delivery) and $21.8 \%$ of cesarean section (274).

Gestational age (GA) at delivery ranged from 38.8 to 39.6 weeks with a mean GA 40 weeks in infected pregnant women while GA at delivery in the non-infected group ranged from 39 to 41 with a mean GA 40 weeks. (p 0.0837).

There was 1 case of antepartum fetal death at term of pregnancy in the group of uninfected women and none in the group of infected women.

\section{Neonatal outcomes}

As for the neonates, 86 neonates from infected pregnant women were delivered during the study period. In infected group birth weight ranged from 3152 to $3349 \mathrm{~g}$ with a mean weight of $3210 \mathrm{~g}$. while in noninfected group mean weight was $3240 \mathrm{~g}(2970-3540)(\mathrm{p}=0.8597)$. Apgar score in infected group at the 5 th minute was 9.64 and in control group was $9.71(p=0.275)$. 8 neonates $(9.5 \%)$ were admitted to the Neonatal Intensive Care Unit (NICU) in infected group and 15 (1.2\%) neonates were admitted to the NICU in non-infected group. Regarding the result of the umbilical artery pH mean, in infected group was 7.30 (7.27-7.30) and in non-innfected group was 7.29 (7.20-7.30) $(p=0.7451)$. 


\section{Discussion}

There is evidence that SARS-CoV-2 can infect syncytiotrophoblast and activate inflammatory responses in placentas of infected women (7) (14).

A recent meta-analysis assessed the impact of COVID 19 pandemic on maternal and perinatal outcomes by comparing the rates of adverse outcomes during the pandemic to those before. This study reported a significant increase in maternal mortality, stillbirth and maternal stress during the pandemic (15)

Hospitalized pregnant women on the day of admission have been adopted universal testing for despite SARS-COV-2 as a standard protocol. High risk pregnant women whose RT-PCR COVID-19 test result was pending were kept in isolation. Breastfeeding was encouraged in all cases, while positive mothers were instructed regarding proper hygiene with their babies.

In our study, we present the results obtained from the total pregnant patients (SARS-COV-2 infected pregnant women and non-infected) that we attended in the period between March 1, 2020, and December 31,2020 ; allowing us to identify specific risk factors associated with adverse maternal and neonatal outcomes.

Regarding sociodemographic factors, no significant differences were found in terms of the mother's age or parity, however, infection by SARS-COV-2 was more frequently observed in not-Hispanic pregnant women, perhaps associated with a lower socioeconomic level that would lead to worse hygienic conditions and overcrowding.

Pre-existing comorbidities such as chronic hypertension, pre-existing diabetes, high maternal age, and high body mass index are risk factors for severe covid-19 in pregnancy (16) (17). In this study we have found a higher incidence of comorbidity in the group of infected pregnant women such as a higher rate of high body mass index, hypertension and pre-gestational diabetes than in the control group, but only diabetes with statistically significant difference $(p=0.0181)$.

On the other hand, our study has demonstrated, in the same way as the studies published so far, that infection by SARS-COV-2 during pregnancy in most cases has a mild clinical course (80.23\%) (18) (19). (asymptomatic 36/86). Furthermore, the rate of severe disease with 7 cases of severe pneumonia (8.1\%) observed in our study is similar to what has been previously reported in other large cohorts and summarized in a recent meta-analysis, in which the risk of severe disease among pregnant women with SARS-COV-2 was estimated to be $13 \%(95 \% \mathrm{Cl} 6-21 \%)$. Although some studies show there is a higher incidence of pregnancy complications in COVID 19 patients, such as fetal growth restriction, gestational diabetes or hypertensive disorders like preeclampsia (20) (21), we did not find them in our study. Moreover, no differences were found in gestational age at delivery, either in the rate of vaginal delivery or cesarean section.

In addition, we did not find any differences in newborn weight, arterial umbilical cord pH or neonatal resuscitation (22) (23). Nevertheless, newborns from infected pregnant women were more likely to be 
admitted to the NICU (9.5\% vs $1.2 \%$ ) than newborns of uninfected mothers. Finally, we did not observe any case of neonatal death.

\section{Conclusions}

Our findings suggest that obstetric patients infected with COVID-19 in our hospital during the first two waves of the pandemic showed a high percentage of asymptomatic patients or with mild symptoms. A higher incidence of maternal comorbidity such as diabetes, obesity and hypertension was observed in the infected group, but without impact on the evolution of the pregnancy, without differences in the gestational age at delivery or the rate of cesarean sections. Only an unexplained increase in neonatal admission to the ICU of neonates of infected mothers was observed.

\section{Declarations}

\section{Acknowledgments}

My thanks to my colleague Dr. Soto Sanchez E.M., true promoter of this work that encourages us to work continuously to improve our clinical practice for pregnant women

\section{Research funding}

None declared.

\section{Author contributions}

All authors have accepted responsibility for the entire content of this manuscript and approved its submission.

\section{Competing interests}

Authors state no conflict of interest.

\section{Informed consent}

Not necessary

\section{Ethical approval}

The local Institutional Review Board deemed the study exempt from review.

\section{References}

1. WHO Coronavirus (COVID-19) Dashboard [Internet]. 2021 [citado 7 de julio de 2021]. Disponible en: https://covid19.who.int 
2. Juan J, Gil MM, Rong Z, Zhang Y, Yang H, Poon LC (2020) Effect of coronavirus disease 2019 (COVID-19) on maternal, perinatal and neonatal outcome: systematic review. Ultrasound Obstet Gynecol 56(1):15-27

3. Kotlyar AM, Grechukhina O, Chen A, Popkhadze S, Grimshaw A, Tal O et al (2021) Vertical transmission of coronavirus disease 2019: a systematic review and meta-analysis. Am J Obstet Gynecol jn 224(1):35-53e3

4. Tolu LB, Ezeh A, Feyissa GT (2021) Vertical transmission of Severe Acute Respiratory Syndrome Coronavirus 2: A scoping review. Luo Z-C. editor PLOS ONE 22 de abril de 16(4):e0250196

5. Carrasco I, Muñoz-Chapuli M, Vigil-Vázquez S, Aguilera-Alonso D, Hernández C, Sánchez-Sánchez C et al (2021) SARS-COV-2 infection in pregnant women and newborns in a Spanish cohort (GESNEOCOVID) during the first wave. BMC Pregnancy Childbirth december 21(1):326

6. Simon E, Cottenet J, Mariet A-S, Bechraoui-Quantin S, Rozenberg P, Gouyon J-B et al Impact of the COVID-19 pandemic on preterm birth and stillbirth: a nationwide, population-based retrospective cohort study.Am JObstet Gynecol. may 2021;S0002937821005639.

7. Bertero L, Borella F, Botta G, Carosso A, Cosma S, Bovetti M et al (2021) ; 1-14

8. Antepartum fetal evaluation: development of a fetal biophysical profile. - ClinicalKey [Internet] (2021) [citado 7 de julio de 2021]. Disponible en: https://www-clinicalkey-es.mhil.a17.csinet.es/\#!/content/medline/2-s2.0-7355965

9. Characteristics of Women of Reproductive Age with Laboratory-Confirmed SARS-CoV-2 Infection by Pregnancy Status - United States, January 22-June 7, 2020. - ClinicalKey [Internet]. 2021 [citado 11 de julio de 2021]. Disponible en: https://www-clinicalkey-es.mhil.a17.csinet.es/\#!/content/medline/2-s2.0-32584795

10. Blasco Santana L, Miraval Wong E, Álvarez-Troncoso J, Sánchez García L, Bartha JL, Regojo-Zapata RM Maternal and perinatal outcomes and placental pathologic examination of 29 SARS-CoV -2 infected patients in the third trimester of gestation.J Obstet Gynaecol Res. 5 de abril de 2021; jog. 14784.

11. Bahadur G, Bhat M, Acharya S, Janga D, Campbell B, Huirne J et al (2021) Retrospective observational RT-PCR analyses on 688 babies born to 843 SARS-CoV-2 positive mothers, placental analyses and diagnostic analyses limitations suggest vertical transmission is possible. Facts Views Vis ObGyn 31 de marzo de 13(1):53-66

12. Harris PA, Taylor R, Thielke R, Payne J, Gonzalez N, Conde JG Research electronic data capture (REDCap)-a metadata-driven methodology and workflow process for providing translational research informatics support.J Biomed Inform. aphril

13. von Elm E, Altman DG, Egger M, Pocock SJ, Gøtzsche PC, Vandenbroucke JP et al (2007) ;147(8):573-7

14. Narang K, Cheek EH, Enninga EAL, Theiler RN (2021) Placental Immune Responses to Viruses: Molecular and Histo-Pathologic Perspectives. Int J Mol Sci 13 de marzo de 22(6):2921 
15. Chmielewska B, Barratt I, Townsend R, Kalafat E, van der Meulen J, Gurol-Urganci I et al (2021) Effects of the COVID-19 pandemic on maternal and perinatal outcomes: a systematic review and meta-analysis. Lancet Glob Health June de 9(6):e759-e772

16. Allotey J, Stallings E, Bonet M, Yap M, Chatterjee S, Kew T et al Clinical manifestations, risk factors, and maternal and perinatal outcomes of coronavirus disease 2019 in pregnancy: living systematic review and meta-analysis.BMJ. 1 de septiembre de 2020;3320.

17. Badr DA, Picone O, Bevilacqua E, Carlin A, Meli F, Sibiude J et al (2021) Severe Acute Respiratory Syndrome Coronavirus 2 and Pregnancy Outcomes According to Gestational Age at Time of Infection. Emerg Infect Dis october 27(10):2535-2543

18. Villar J, Ariff S, Gunier RB, Thiruvengadam R, Rauch S, Kholin A et al (2021) Maternal and Neonatal Morbidity and Mortality Among Pregnant Women With and Without COVID-19 Infection: The INTERCOVID Multinational Cohort Study. JAMA Pediatr 1 de agosto de 175(8):817

19. Antsaklis P, Tasias K, Psarris A, Theodora M, Daskalakis G, Rodolakis A (2021) Covid-19 and pregnancy: the experience of a tertiary maternity hospital. J Perinat Med 27 de julio de 49(6):686690

20. Conde-Agudelo A, Villar J, Lindheimer M (2008) Maternal infection and risk of preeclampsia: systematic review and metaanalysis. Am J Obstet Gynecol january 198(1):7-22

21. Vouga M, Favre G, Martinez-Perez O, Pomar L, Acebal LF, Abascal-Saiz A et al (2021) Maternal outcomes and risk factors for COVID-19 severity among pregnant women. Sci Rep 6 de julio de 11(1):13898

22. Pountoukidou A, Potamiti-Komi M, Sarri V, Papapanou M, Routsi E, Tsiatsiani AM et al Management and Prevention of COVID-19 in Pregnancy and Pandemic Obstetric Care: A Review of Current Practices. Healthcare. 15 de abril de 2021; 9(4):467

23. Cruz Melguizo S, de la Cruz Conty ML, Carmona Payán P, Abascal-Saiz A, Pintando Recarte P, González Rodríguez L et al (2021) Pregnancy Outcomes and SARS-CoV-2 Infection: The Spanish Obstetric Emergency Group Study. Viruses mayo de 13(5):853

\section{Tables}


Table 1

Demographic features and clinical characteristics of for SARS-CoV-2 positive cases $(n=86)$ and SARSCoV.2 negative controls ( $\mathrm{n}=1282$ ).

\begin{tabular}{|c|c|c|c|}
\hline Variables & $\begin{array}{l}\text { Infected group } \\
(n=86)\end{array}$ & $\begin{array}{l}\text { Non-infected group } \\
(n=1262)\end{array}$ & V- \\
\hline \multicolumn{4}{|l|}{ Maternal Characteristics } \\
\hline $\begin{array}{l}\text { Maternal age, years (mean, } 95 \% \\
\mathrm{Cl} \text { ) }\end{array}$ & $30(28.36-31,20)$ & $31,50(26-36)$ & 0,048 \\
\hline \multicolumn{4}{|l|}{ Parity, $(\mathrm{n}, \%)$} \\
\hline Nulliparous (n, \%) & $41(48,24 \%)$ & $548(43.44 \%)$ & 0.121 \\
\hline Multiparous (n, \%) & $45(51.77 \%)$ & $714(55,58 \%)$ & 0,112 \\
\hline \multicolumn{4}{|l|}{ Ethnicity } \\
\hline Hispanic (n, \%) & $29(34.52 \%)$ & $605(51.9 \%)$ & \\
\hline Other countries $(\mathrm{n}, \%)$ & $55(64,48 \%)$ & $654(48,05 \%)$ & 0,002 \\
\hline No comorbidities (n, \%) & $123(27.58 \%)$ & $59(68.60 \%)$ & 0,002 \\
\hline \multicolumn{4}{|l|}{ Maternal comorbidities } \\
\hline Thyroid disfunction (n, \%) & $4(4.64 \%)$ & $12(0.95 \%)$ & 0.004 \\
\hline Pulmonary comorbidities (n, \%) & $1(1.16 \%)$ & $5(0.39 \%)$ & 0.061 \\
\hline Hematologic comorbidities (n, \%) & $2(2.32 \%)$ & $13(0.85 \%)$ & 0.082 \\
\hline Obesiity $(\mathrm{IBM} \geq 30)(\mathrm{n}, \%)$ & $9(10.47 \%)$ & $80(6.34 \%)$ & 0.172 \\
\hline Hypertension disease (n, \%) & $4(4.65 \%)$ & $22(1.58 \%)$ & 0.079 \\
\hline Pregestational diabetes $(n, \%)$ & $5(5.81 \%)$ & $20(1.58 \%)$ & 0.018 \\
\hline
\end{tabular}


Table 2

Clinical characteristics of SARS-CoV-2 positive cases.

\begin{tabular}{|ll|}
\hline Variables & Infected group $n=86$ \\
\hline Gestational age at diagnosis, weeks (mean, 95\% Cl) & $38(36.05-37.77)$ \\
\hline Clinical manifestation & $37(43.02 \%)$ \\
\hline Asymptomatic & $49(56.98 \%)$ \\
\hline Symptomatic $(n, \%)$ & $69(80.23 \%)$ \\
\hline Mild $(n, \%)$ & $10(11.63 \%)$ \\
\hline Moderate $(n, \%)$ & $7(8.14 \%)$ \\
\hline Severe $(n, \%)$ & $7(8.14 \%)$ \\
\hline Pneumonia $(n, \%)$ & $31(36.05 \%)$ \\
\hline Fever $(n, \%)$ & $100(100 \%)$ \\
\hline Ageusia $(n, \%)$ & $10(11.63 \%)$ \\
\hline Myalgia $(n, \%)$ & $14(16.28 \%)$ \\
\hline Headache $(n, \%)$ & $1(1.16 \%)$ \\
\hline Sore throat $(n, \%)$ & $18(20.93 \%)$ \\
\hline Dyspnea $(n, \%)$ & $15(15.58-17.74)$ \\
\hline Covid infection duration (days, median IRC 95\%) (n, \%) & $14(16.67 \%)$ \\
\hline Pulomary maduration needed (betamethisone) & $1(1.18 \%)$ \\
\hline Maternal ICU admision (n, \%) & \\
\hline ICU, intensive care unit. & \\
\hline
\end{tabular}


Table 3

Obstetric and neonatal outcomes of SARS-COV-2 positive cases $(\mathrm{n}=86)$ and SARS-CoV- 2 negative controls ( $n=1282)$.

\begin{tabular}{|c|c|c|c|}
\hline Variables & $\begin{array}{l}\text { Infected group } \\
(n=86)\end{array}$ & $\begin{array}{l}\text { Non-infected group } \\
(n=1262)\end{array}$ & $\begin{array}{l}\text { p- } \\
\text { Value }\end{array}$ \\
\hline \multicolumn{4}{|l|}{ Pregnancy complications } \\
\hline FGR $(n, \%)$ & 0 & $13(1.01 \%)$ & 0.000 \\
\hline GDM (n, \%) & 0 & $14(1.09 \%)$ & 0.000 \\
\hline Pre-eclampsia (n, \%) & 0 & $3(0.23 \%)$ & 0.253 \\
\hline Placenta accreta/previa (n, \%) & 0 & 2 & 0.245 \\
\hline $\begin{array}{l}\text { Pulmonary maduration nedeed with } \\
\text { betamethisone }(n, \%)\end{array}$ & $14(16.67 \%)$ & $14(1.11 \%)$ & 0.000 \\
\hline Vaginal delivery $(\mathrm{n}, \%)$ & $70(83,34 \%)$ & $983 / 77,89 \%)$ & 0.366 \\
\hline Cesarean section $(n, \%)$ & $14(16,67 \%)$ & $274(21,71 \%)$ & 0.362 \\
\hline $\begin{array}{l}\text { Gestational age at delivery, weeks (median, } \\
\text { ICR } 95 \%)(n, \%)\end{array}$ & $40(38,84-39,56)$ & $40(39-41)$ & 0.084 \\
\hline Maternal admission to ICU (n, \%) & $1(1.18 \%)$ & $5(0.40 \%)$ & 0.325 \\
\hline Fetal distress (n, \%) & $13(15.12 \%)$ & $130(10.34 \%)$ & 0.203 \\
\hline Delivery complications (n, \%) & $10(11.90 \%)$ & $205(16.28 \%)$ & 0.357 \\
\hline Birth weight, g. (median, ICR 95\%) (n, \%) & $\begin{array}{l}3210(3152- \\
3349)\end{array}$ & 3240 (2970-3540) & 0.859 \\
\hline \multirow[t]{3}{*}{ Apgar score 1 st $\min \geq 8(n, \%)$} & $79(91 \%)$ & $1148(92.4 \%)$ & \multirow[t]{3}{*}{0.301} \\
\hline & Median 9 (9-9) & Median 9 (8-9 & \\
\hline & Mean 8.59 & Mean 8.57 & \\
\hline \multirow[t]{2}{*}{ Apgar score 5th $\min \geq 8(\mathrm{n}, \%)$} & $85(98,84 \%)$ & \multirow{2}{*}{$\begin{array}{l}1233 \text { Median } 10 \text { (10- } \\
\text { 10) }\end{array}$} & \multirow[t]{2}{*}{0.275} \\
\hline & $\begin{array}{l}\text { Median } 10(10- \\
\text { 10) }\end{array}$ & & \\
\hline Stillbirth (n, \%) & 0 & $1(1,16 \%)$ & 0.356 \\
\hline Umbilical artery pH (n, \%) & $7,30(7,27-7,30)$ & $7,29(7,20-7,30)$ & 0.745 \\
\hline Neonatal admission to NICU & $8(9,52 \%)$ & $15(1.19 \%)$ & 0.000 \\
\hline
\end{tabular}

